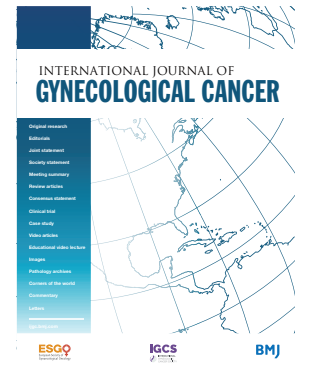

Department of Radiation Oncology, Comprehensive Cancer Center, Medical University of Vienna, Vienna, Austria

Correspondence to Dr Alina Emiliana Sturdza, Department of Radiation Oncology, Comprehensive Cancer Center, Medical University of Vienna, Vienna 1090, Austria; alina.sturdza@ meduniwien.ac.at

Received 20 October 2021 Accepted 14 December 2021

\section{Check for updates}

(C) IGCS and ESGO 2022. No commercial re-use. See rights and permissions. Published by BMJ.

To cite: Sturdza AE, Knoth J. Int J Gynecol Cancer

2022;32:273-280.

\title{
Image-guided brachytherapy in cervical cancer including fractionation
}

Alina Emiliana Sturdza, Johannes Knoth

\begin{abstract}
Image-guided brachytherapy in cervical cancer has been developed to be a feasible and very efficient component of the treatment of locally advanced cervical cancer in addition to concurrent chemoradiation treatment. This technique allows effective dose coverage of the target while sparing the organs at risk through adjustment of the implants (intracavitary and interstitial needles) and multi-pararametric three-dimensional treatment planning. Emerging evidence from prospective studies shows a high rate of local control throughout all stages, superior to two-dimensional brachytherapy, with limited toxicity for each organ site. This is associated with a high rate of pelvic control and overall survival. Based on clinical evidence, there is a dose-effect relationship for both disease and morbidity endpoints from which clear dose constraints for the target and organs at risk were derived. This review gives an overview of the major milestones that occurred in the development of image-guided adaptive brachytherapy in the last two decades, including outcome data and a summary of the hard and soft dose constraints recommended for targets and organs at risk.
\end{abstract}

\section{INTRODUCTION}

Globally, cervical cancer is still a challenge. Despite screening, vaccination and cutting-edge technology treatments being available, there are still women dying with this disease in many parts of the world. One of the main aims of the gynecological and governmental societies in the new millennium is for everyone to have access to state-of-the-art prevention and treatments for cervical cancer. ${ }^{1}$

Radiation oncology treatments are the main stay in locally advanced cervical cancer when curative intention is the goal. After concurrent external beam radiation therapy and chemotherapy, a very focused, volume-prescribed radiation dose is delivered to the tumor rest in the cervix through a technique called brachytherapy. Brachytherapy is an essential component of locally advanced cervical cancer treatment and plays a critical role. Multiple studies have demonstrated a decrease in local recurrence and an improvement in overall survival when brachytherapy is used in these patients. ${ }^{2-4}$ The success of brachytherapy requires extreme conformity, with delivery of a high radiation dose directly to the tumor while sparing surrounding normal tissues via rapid radiation dose fall-off beyond the implanted tumor volume. In the past, brachytherapy was performed based on two-dimensional images and dose prescription to a reference point: point $A$. This was originally defined as a point located $2 \mathrm{~cm}$ superior to the lateral vaginal fornix and $2 \mathrm{~cm}$ lateral to the cervical canal, assuming that the region represented the tolerance limits due to crossing of the uterine artery and ureter. In the last 20 years of the twentieth century, the importance of better imaging shifted the drive of research toward better modalities of guidance in brachytherapy. The initial steps were made by CT-guided brachytherapy, ${ }^{5}$ followed by MRI guidance being either incorporated or as a unique imaging modality. ${ }^{67}$ Often transabdominal or transrectal ultrasound guidance is also used to supplement the information about the tumor rest, especially during the applicator insertion and in some institutions for planning purposes. ${ }^{8-11}$ In a few centers positron emission tomographie (PET)-CT has been used in clinical practice for brachytherapy guidance purposes. ${ }^{12}$ The overall aim of this paper is to review and describe the current status of image-guided brachytherapy for cervical cancer.

\section{TARGET VOLUME AND ORGAN AT RISK DEFINITION AND DOSE PRESCRIPTION, REPORTING, AND FRACTIONATION}

Through the advent of image guidance, both the target and the organs at risk were better visualized and a whole era of developments in brachytherapy has elapsed. Precise target and organ at risk delineation were determined and incorporated in recommendations for use in clinical practice. ${ }^{13-16}$ MRI has been the imaging modality of first choice for the female pelvis since the early 1990s due to its intrinsic high soft tissue contrast. Therefore, the vast majority of the dramatic technical advances that occurred in the brachytherapy of gynecological malignancies are based on MRI procedures. The newly introduced adaptive approach was based on the assumption that volumes containing microscopic disease at diagnosis can be controlled with external chemoradiation of 45-50 Gy, whereas the brachytherapy boost aims to control the following: volumes that contain residual macroscopic tumor or assumed pathological tissue at the time of brachytherapy, and volumes with potential residual microscopic disease in the region where the tumor was present at diagnosis. ${ }^{17}$ Consequently, the residual gross tumor volume as seen on MRI is 


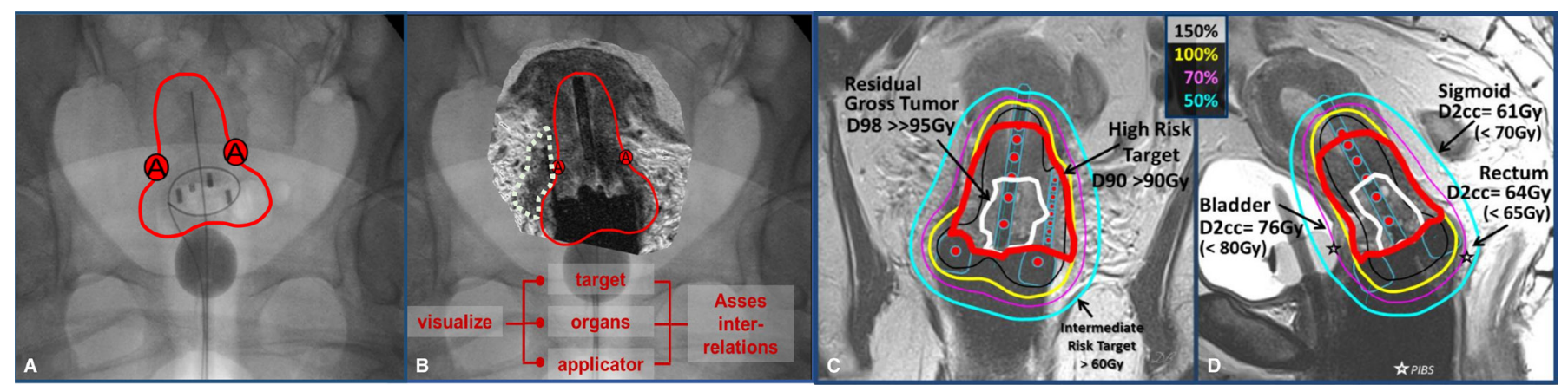

Figure 1 Milestones in the development of brachytherapy in locally advanced cervical cancer from X-ray-based (twodimensional) to MRI-guided adaptive brachytherapy. (A) Two-dimensional brachytherapy using radiography as imaging modality and standard plans with dose prescription to point A (situated $2 \mathrm{~cm}$ superior to the lateral vaginal fornix and $2 \mathrm{~cm}$ lateral to the cervical canal) irrespective of tumor size and response to chemoradiation. (B) MRI of a patient with locally advanced cervical cancer with applicator in situ superimposed on the X-ray of the same patient and a standard twodimensional plan prescribed to point $A$. The dotted white line shows a rest tumor in the right parametria which could not be covered by the prescription dose of a standard plan, increasing the risk of local recurrence in this patient. (C) Coronal view of a T2-weighted MRI of a patient with locally advanced cervical cancer with rest tumor in the left parametria for whom an intracavitary/interstitial tandem and ring applicator with interstitial needles on the left was performed. Delineated volumes: residual gross tumor volume (GTV-T $\mathrm{T}_{\text {res }}$ ) is encompassed by the $150 \%$ isodose line (in black) by a D98>>95 Gy with D98 being the near minimum dose encompassing that volume; the adaptive high-risk clinical target volume $\left(\right.$ CTV-T $\left.{ }_{H R}\right)$ encompassed by the $100 \%$ prescription dose (yellow) resulting in a dose to $90 \%$ of the volume of $>90$ Gy; the adaptive intermediate-risk target volume (CTV-T ) encompassed by the $50 \%$ prescribed dose resulting in a dose to $90 \%$ of this volume of $>60 \mathrm{~Gy}$ (according to EMBRACE II protocol). (D) Sagittal view of a T2-weighted MRI of the same patient as in (C), depicting the minimum dose to the most exposed $2 \mathrm{~cm}^{3}\left(\mathrm{D} 2 \mathrm{~cm}^{3}\right)$ of the bladder wall which in this case is $<80 \mathrm{~Gy}, \mathrm{D} 2 \mathrm{~cm}^{3}$ rectal wall which is $64 \mathrm{~Gy}$, and D2cm ${ }^{3}$ for the sigmoid wall which is 61 Gy (according to EMBRACE II protocol). ${ }^{24}$

delineated at the time of brachytherapy. The adaptive high-risk clinical target volume incorporating the residual gross tumor volume, the whole cervix, and areas still at risk for harboring microscopic disease is contoured. The high-risk clinical target volume became an important parameter for dose prescription ${ }^{18}$ and later was discovered to be a prognostic factor for overall survival. ${ }^{19} \mathrm{~A}$ third target volume, the adaptive intermediate risk clinical target volume, takes into account the tumor size at the time of diagnosis, or when applicable, a safety margin added to the high-risk clinical target volume. More recently, these volume definitions based on MRI with applicator in situ at the time of brachytherapy were adapted for CT guidance to be used in centers in which MRI is not available at brachytherapy, but instead CT is used. ${ }^{15}$ Briefly, the minimum requirements are clinical examination and documentation, CT or MRI at diagnosis, and at minimum CT at the time of brachytherapy. CT-based target contouring recommendations are based on four tumor remission categories within eight defined environments that are described in detail in the recommendations. ${ }^{15}$ The International Commission on Radiation Units \& Measurements 89 report gives recommendations on contouring of organs at risk on threedimensional images with the brachytherapy applicator in situ. ${ }^{16}$ These recommendations should include contouring of the outer wall of the bladder, rectum, sigmoid colon, and bowel.

As a natural development, the dose prescription shifted gradually from the classical point A to the volumes described above. Individual plans were created in order to adapt the $100 \%$ prescribed dose to the volume of the rest tumor at the time of brachytherapy, rather than using standard plans, irrespective of tumor size $e^{16} 18$ (Figure 1). Recommendations for dose and volume reporting of three-dimensional image-guided brachytherapy in locally advanced cervical cancer are outlined in the International Commission on
Radiation Units \& Measurements 89 report and include the summed dose from external beam radiation and brachytherapy boost. ${ }^{16}$ The current standard for reporting the equi-effective dose in cervical cancer image-guided adaptive brachytherapy is an equivalent dose in 2 Gy fractions (EQD2) using alpha/beta ratios of $10 \mathrm{~Gy}$ for tumor volumes and $3 \mathrm{~Gy}$ for organs at risk. ${ }^{16}$ For pulsed dose rate brachytherapy, a repair half time of 1.5 hours is the current standard. The principles presented here are based on schedules of 45-50 Gy external beam radiation to the whole pelvis and $40-45$ Gy of brachytherapy either applied by high dose rate or pulsed dose rate. To obtain and maintain local control, low-dose regions in the target volumes are assumed critical, and reporting of the near minimum dose (D98) for all contoured volumes is recommended. ${ }^{17}$ One very important dose volume histogram parameter is the dose delivered to $90 \%$ of the high-risk clinical target volume, which is representative of a large portion of the target. ${ }^{18}$ The median dose (D50) reflects the high dose delivered to the central part of the highrisk clinical target volume, which may presumably also have importance for local control. ${ }^{17}$ Regarding the reporting of dose volume histogram parameters for organs at risk to assess morbidity, it was shown that the high-dose regions are essential. Dose to hotspots in the organ walls (for the bladder, rectum, sigmoid colon, and bowel) are best described through reporting the dose as small absolute volume, such as $0.1 \mathrm{~cm}^{3}\left(\mathrm{D} 0.1 \mathrm{~cm}^{3}\right)$ and $2 \mathrm{~cm}^{3}\left(\mathrm{D} 2 \mathrm{~cm}^{3}\right)$. Additionally, the International Commission on Radiation Units \& Measurements bladder point dose and rectovaginal point dose are correlated to bladder and vaginal long-term toxicity; therefore, they should also be reported. ${ }^{20}{ }^{21}$ The Commission's rectovaginal reference point is positioned at the intersection level between the tandem and the source positions in the ovoids or ring and $5 \mathrm{~mm}$ dorsal of the posterior vaginal wall on the axis perpendicular to the body axis. The 
dose to the rectovaginal point has been shown to correlate with the probability of developing vaginal stenosis. ${ }^{20}$

Administration of higher external beam radiation doses leads to considerably larger volumes being irradiated to intermediate dose levels, with no benefit to local control, but rather increased toxicity. ${ }^{22}{ }^{23}$ Therefore, it can be considered beneficial to restrict the external beam radiation dose to $45 \mathrm{~Gy}$. In the case of pathologically enlarged lymph nodes, a dose escalation of $55 \mathrm{~Gy}$ to the pelvic lymph nodes and 57-60 Gy to the para-aortic lymph nodes could be applied through a sequential or a simultaneously integrated boost. Schedules with $45 \mathrm{~Gy}$ external beam radiation require larger brachytherapy dose contribution, potentially more brachytherapy fractions, and higher demands on the brachytherapy quality (such as the availability of intracavitary/interstitial implants). ${ }^{17}$ It is recommended that brachytherapy be applied during weeks $6-7$ of treatment. ${ }^{24} \mathrm{~A}$ frequently used fractionation schedule for imageguided adaptive brachytherapy includes 45 Gy external beam radiation combined with four fractions of image-guided adaptive brachytherapy, which may be delivered in two applicator insertions. In this case, a full recovery is assumed to occur between fractions, therefore an interval of at least 6-8 hours is recommended between fractions. ${ }^{16}$

Reduction from four to two or three fractions may be feasible for small tumors or tumors with good response by applying sufficient dose to the limited size high-risk clinical target volume, without violating organ at risk dose constraints. Large residual tumor volumes with large size, high-risk clinical target volume will benefit from four image-guided adaptive brachytherapy fractions to achieve the highest possible tumor control with acceptable morbidity. ${ }^{17} \mathrm{~A}$ large number of centers in which MRI-based image-guided adaptive brachytherapy is performed are delivering pulsed dose rate brachytherapy in one, or two to three applications, in general with 10-45 pulses/fraction and one pulse/hour delivered. Regardless of the dose rate (high dose rate or pulsed dose rate), it is essential that a dose to $90 \%$ of the high-risk clinical target volume receives a minimum dose of $85 \mathrm{~Gy} \mathrm{EQD}$, within an overall treatment time of $45-50$ days. ${ }^{25}$ Data regarding delivery of chemotherapy during brachytherapy are scarce. A monoinstitutional retrospective study looking at patients treated for locally advanced cervical cancer including pulsed dose rate image-guided adaptive brachytherapy showed that an additional cycle should be considered at the time of brachytherapy if the patient's blood cell count is within a normal range, especially in patients with large, high-risk clinical target volume at the time of brachytherapy. ${ }^{26}$

Based on clinical evidence, ${ }^{25}$ a dose of $>85$ Gy (dose to $90 \%$ of the high-risk clinical target volume) delivered in 7 weeks provides 3 year local control rates of $>94 \%$ in limited size high-risk clinical target volume $\left(20 \mathrm{~cm}^{3}\right),>93 \%$ in intermediate size $\left(30 \mathrm{~cm}^{3}\right)$, and $>86 \%$ in large size $\left(70 \mathrm{~cm}^{3}\right)$ high-risk clinical target volume. For intermediate-risk clinical target volume, residual gross tumor volume dose of $>60$ Gy and $>95$ Gy (D98) leads to similar local control. A dose of $5 \mathrm{~Gy}$ (to $90 \%$ of the high-risk clinical target volume) is required to compensate for an increase in overall treatment time by 1 week. ${ }^{25}$ The dose constraints and levels of local control introduced in the EMBRACE II protocol are based on these data, and therefore if treatment time is longer or shorter than 7 weeks, the dose planning aims should be adjusted by 5 Gy per week for highrisk clinical target volume. The data underline the importance of keeping the overall treatment time as short as possible, especially for large size high-risk clinical target volume, where a higher dose is needed to reach $>90 \%$ local control. In any case, every effort should be made to keep the overall treatment time $<50$ days. ${ }^{24}$ Current common schedules such as 45 Gy external beam radiotherapy combined with image-guided adaptive brachytherapy of two fractions of $9 \mathrm{~Gy}$, five fractions of $5.5 \mathrm{~Gy}$, or four fractions of $7 \mathrm{~Gy}$ correspond to a total equivalent dose in $2 \mathrm{~Gy}$ fractions using $\mathrm{a} / \mathrm{b}=10\left(\mathrm{EQD2}{ }_{10}\right)$ of $73 \mathrm{~Gy}, 80 \mathrm{~Gy}$, and $84 \mathrm{~Gy}$, respectively $(\mathrm{a} / \mathrm{b}=10)$.

Very recently, a prospective study showed that delivering three fractions with one application is feasible and results in a dose of $90 \%$ of the high-risk clinical target volume of over $84 \mathrm{~Gy}$ when brachytherapy is performed in a specialized center. ${ }^{27}$ Based on recent clinical evidence from RetroEMBRACE and EMBRACE I and some monoinstitutional studies, specific dose prescription recommendations for the target and constraints for organs at risk are currently available (Table 1). ${ }^{1725}$

In order to respect the planning aims, more complex applications including intracavitary/interstitial implants may be required. Although it is an invasive procedure, operator dependent (similar to surgical procedures), and requires a learning period, there is an obvious increase in the number of interstitial procedures in cervical cancer from $23 \%{ }^{28}$ to $43 \%{ }^{29}$ and $70 \%$ in EMBRACE II. Contrary to expectations, intracavitary/interstitial implants do not result in more long-term side effects, but they do dramatically improve local control, especially in large and/or asymmetrical tumors at the time of brachytherapy. ${ }^{30}{ }^{31}$ In parallel to imaging development as a guidance tool for brachytherapy, different types of MRI and CT compatible applicators were developed. These allow insertion of the needles to cover the parametrial infiltration to the pelvic wall and vaginal involvement. ${ }^{32-35}$ In some cases, free or transperineal application of needles could be required (Figure 2). Some implantation-related side effects are reported in the literature with the use of combined intracavitary/interstitial applicators. The most feared acute complication is significant bleeding. In a cohort of 69 patients with locally advanced cervical cancer and large tumor rest at the time of brachytherapy with infiltration of parametria \pm pelvic wall, in whom Vienna II tandem/ring and titan needles were inserted, acute bleeding occurred at a relatively high rate. ${ }^{34}$ During applicator removal, active bleeding was observed in 19 patients $(27.5 \%)$. These events were classified as arterial in six patients and were seen mainly with a large diameter ring ( 30 or $34 \mathrm{~mm}$ ), venous in 12 patients, and arterial bleeding due to myoma rupture occurred in one patient. In a different cohort of 61 patients including all stages of locally advanced cervical cancer who were treated by means of a hybrid applicator (Venezia), significant bleeding also occurred after removal of the applicator, but only in four of 241 applications. ${ }^{35}$ This low rate may be due to improved ease of insertion/removal of the split ring design of the Venezia applicator compared with the standard ring. Nonetheless, in both papers, arterial or venous bleeding was conservatively managed by the radiation oncology team, with continuous compression for at least 6-10 min. The patient with the myoma rupture underwent surgical intervention and was discharged later. Regardless of the application type, intracavitary/interstitial implants with image guidance allows target coverage in tumors that in the past were deemed untreatable. 


\section{Review}

Table 1 Summary of dose-effect and volume-effect relationships for endpoints relevant for brachytherapy and the currently recommended soft and hard constraints (modified according to Tanderup et $\mathrm{al}^{17}$ ). The table is based on an update of the EMBRACE II constraints which are found in the EMBRACE II protocol ${ }^{24}$

\begin{tabular}{|c|c|c|c|c|c|}
\hline \multirow{2}{*}{$\begin{array}{l}\text { Target/organ } \\
\text { at risk }\end{array}$} & \multirow[b]{2}{*}{ Dose } & \multirow[b]{2}{*}{ Endpoint } & \multicolumn{2}{|c|}{ Recommended dose planning aims } & \multirow{2}{*}{$\begin{array}{l}\text { Level of } \\
\text { evidence }\end{array}$} \\
\hline & & & Soft constraint & Hard constraint & \\
\hline $\begin{array}{l}\text { High-risk } \\
\text { clinical target } \\
\text { volume }\end{array}$ & $\begin{array}{l}\text { Dose to } 90 \% \text { of the } \\
\text { volume }\end{array}$ & Local control & $\begin{array}{l}>90 \text { Gy EQD2 }{ }_{10} \\
<95 \text { Gy EQD2 } 10\end{array}$ & $>85$ gy EQD2 ${ }_{10}$ & High \\
\hline $\begin{array}{l}\text { High-risk } \\
\text { clinical target } \\
\text { volume }\end{array}$ & D98 & Local control & >80 Gy EQD2 10 & >75 Gy EQD2 ${ }_{10}$ & \\
\hline Bladder & $\mathrm{D} 2 \mathrm{~cm} 3$ & $\begin{array}{l}\text { Bleeding, cystitis, } \\
\text { fistula }\end{array}$ & $<80$ Gy EQD2 3 & $<85$ Gy EQD2 3 & High \\
\hline Bladder & $\begin{array}{l}\text { International Commission } \\
\text { on Radiation Units \& } \\
\text { Measurements bladder } \\
\text { point }\end{array}$ & Incontinence & $<75$ Gy EQD2 3 & $<85$ Gy EQD2 3 & High \\
\hline Sigmoid & $\mathrm{D} 2 \mathrm{~cm} 3$ & $\begin{array}{l}\text { Diarrhea, fistula, } \\
\text { strictures, bleeding }\end{array}$ & $<70$ Gy EQD2 3 & $<75$ Gy EQD2 3 & $\begin{array}{l}\text { No correlation/ } \\
\text { under } \\
\text { investigation }\end{array}$ \\
\hline Bowel & $\mathrm{D} 2 \mathrm{~cm} 3$ & $\begin{array}{l}\text { Fistula, strictures, } \\
\text { incontinence, } \\
\text { bleeding }\end{array}$ & $<65$ Gy EQD2 3 & $<75$ Gy EQD2 3 & High \\
\hline Vagina & $\begin{array}{l}\text { International Commission } \\
\text { on Radiation Units } \\
\text { \& Measurements } \\
\text { rectovaginal point }\end{array}$ & Vaginal stenosis & $<65$ Gy EQD2 3 & $<75$ Gy EQD2 3 & High \\
\hline
\end{tabular}

D98, dose to $98 \%$ of volume; D2cm3, minimum dose to the most exposed $2 \mathrm{~cm}^{3}$ of organ wall; EQD2 ${ }_{3}$, equivalent dose in 2 Gy fractions (EQD2) using alpha/beta ratios of 3 Gy for organs at risk; EQD2 ${ }_{10}$, equivalent dose in 2 Gy fractions (EQD2) using alpha/beta ratios of 10 Gy for tumour volumes; high-risk clinical target volume, adaptive high-risk clinical target volume.

\section{OUTCOME}

\section{Target}

From the early 1990s when the first image-guided adaptive brachytherapy was attempted in locally advanced cervical cancer, to the present time, a large number of centers in the world have incorporated this technique in clinical practice. Several singlecenter series, ${ }^{67223637}$ a non-randomized prospective French study using CT guidance, ${ }^{30}$ and a large retrospective multicenter series ${ }^{28}$ using mainly MRI guidance have shown the safety and feasibility of image-guided adaptive brachytherapy in routine practice with clinically and statistically significant improved local and pelvic control and reduced morbidity. Most recently, the first large-scale multiinstitutional prospective study (24 institutions) using MRI guidance of brachytherapy confirmed the findings conveyed in the previous papers. ${ }^{29}$ The study reported at a median follow-up of 51 months an actuarial 5 year local control of $92 \%(95 \% \mathrm{Cl} 90 \%$ to $93 \%)$, pelvic control of $87 \%$ ( $85 \%$ to $89 \%)$, overall survival of $74 \%(72 \%$ to $77 \%$ ), and disease-free survival of $68 \%$. Actuarial cumulative 5 year incidence of grade $3-5$ morbidity was $6.8 \%$ (95\% $\mathrm{Cl} 5.4 \%$ to $8.6 \%$ ) for genitourinary events, $8.5 \%$ (6.9\% to $10.6 \%$ ) for gastrointestinal events, $5.7 \%$ (4.3\% to $7.6 \%)$ for vaginal events, and $3.2 \%(2.2 \%$ to $4.5 \%)$ for fistulae.

The treatment consisted of chemoradiotherapy (weekly intravenous cisplatin $40 \mathrm{mg} / \mathrm{m}^{2}$, five to six cycles, 1 day per cycle, plus 45-50 Gy external-beam radiotherapy delivered in 1.8-2 Gy fractions) followed by MRI-based image-guided adaptive brachytherapy. The MRI-based image-guided adaptive brachytherapy target volume definition and dose reporting were according to Group de Curiethérapie-European SocieTy for Radiotherapy \& Oncology recommendations. Image-guided adaptive brachytherapy dose prescription was open according to institutional practice. ${ }^{29}$ In this cohort, 1317 patients underwent image-guided adaptive brachytherapy including dose optimization for a median high-risk clinical target volume of $28 \mathrm{~cm}^{3}$ (IQR 20-40) and median dose to $90 \%$ of the high-risk clinical target volume was 90 Gy (IQR 85-94) EQD2. The study accrued patients between 2008 and 2015, therefore FIGO 2009 staging was used. Actuarial stage-based local control and overall survival were reported as follows: stage IB1: 

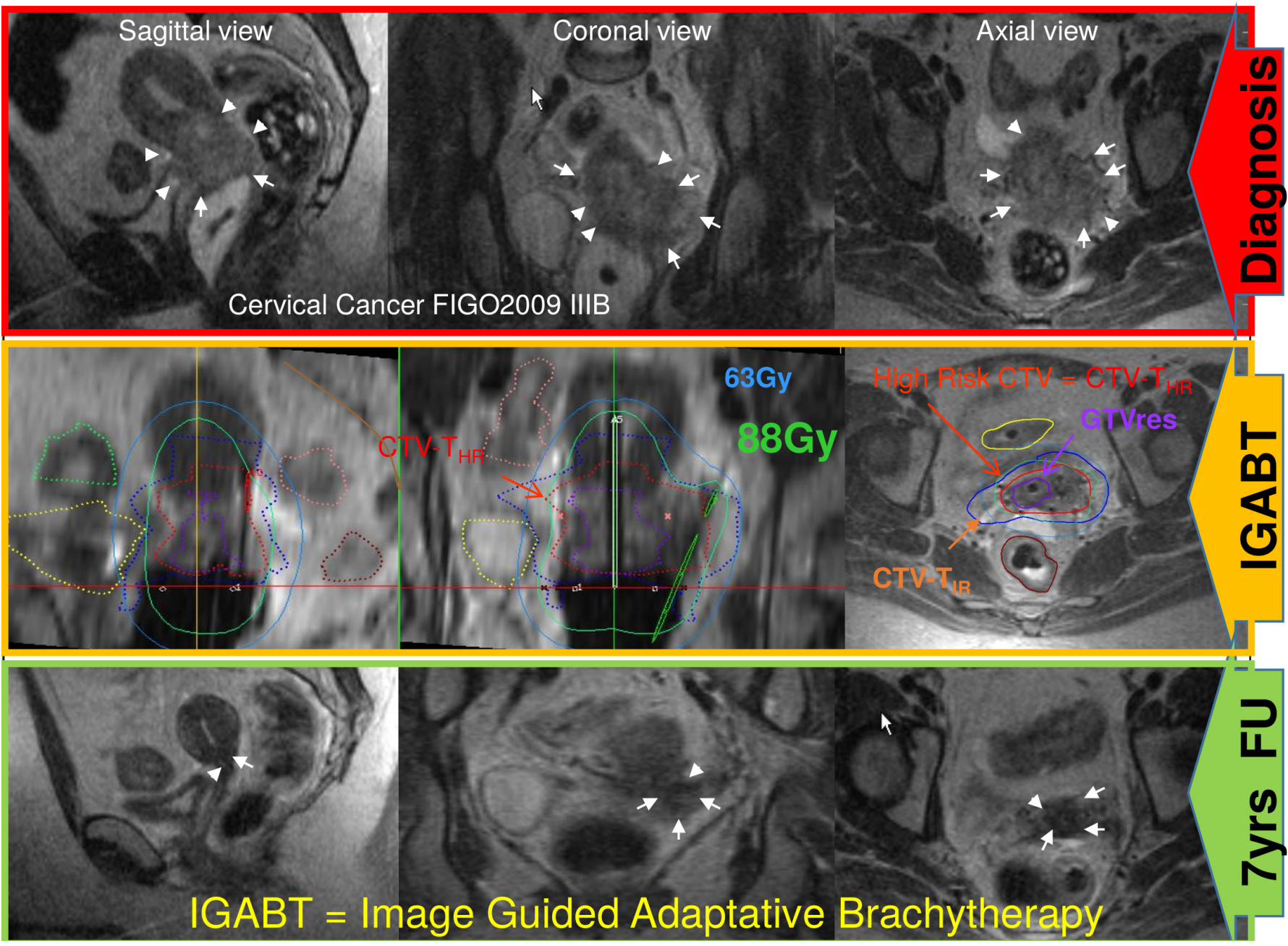

Figure 2 Rationale for interstitial application. In this example of left parametrial involvement to the pelvic wall at the time of diagnosis and residual tumor to the left middle parametria at the time of IGABT, a dose of 88 Gy to the D90 CTV-T possible through straight and oblique needles inserted through the ring applicator. T2-weighted MRI sagittal, coronal, and axial images were taken at the time of diagnosis, IGABT, and after 7 years of follow-up (patients remained in complete remission). $\mathrm{CTV}_{\mathrm{IR}}$, intermediate-risk clinical target volume; GTV-T ${ }_{\text {res, }}$, residual gross tumor volume; IGABT, image-guided adaptive brachytherapy.

$98 \%$ and $83 \%$; stage IB2: $92 \%$ and $73 \%$; stage IIA1: $91 \%$ and $80 \%$; stage IIA2: $89 \%$ and $74 \%$; stage IIB: $91 \%$ and $78 \%$; stage IIIA: $100 \%$ and $76 \%$; stage IIIB: $92 \%$ and $64 \%$; stage IVA; $91 \%$ and $52 \%$; stage IVB based on para-aortic lymph node involvement $89 \%$ and $61 \%$. Throughout the stages, local control in this study is superior to historical series using two-dimensional point $A$ based brachytherapy. ${ }^{23} 3839$ This improvement is remarkable (over 14\%) in stage IIIB disease. These excellent local control rates are probably due to improvement in target contouring, implant technique, and three-dimensional planning.

\section{Organs at Risk}

Side effects in patients treated definitively for locally advanced cervical cancer are related to both external beam radiation and image-guided adaptive brachytherapy. They are patient reported and physician reported, and therefore are prone to report bias, are interconnected, and it may be difficult to differentiate between organ levels, but they impact to an important degree on the quality of life of patients. Severe grade $3-5$ toxicity occurs within 90 days of completing the treatment and actuarial incidence increases slowly after 3-5years. ${ }^{40}$ The prospective STIC trial showed that three-dimensional brachytherapy results in less late severe toxicity incidence in comparison to two-dimensional brachytherapy. ${ }^{30}$

The image-guided adaptive brachytherapy experience has also provided descriptive evaluations of morbidity time patterns as well as analyses of risk factors, including dose. In the multi-institutional cohort of EMBRACE I, ${ }^{29}$ a total of 330 grade 3-5 morbidity events (genitourinary, gastrointestinal, vaginal, fistulas) were observed in $183(14.6 \%)$ of 1251 patients. The most common grade 3-5 events (5year actuarial cumulative incidence) were fistulae $(3.2 \%, 95 \% \mathrm{Cl}$ $2.2 \%$ to $4.5 \%$; 42 events), gastrointestinal stenosis $(2.8 \%, 1.9 \%$ to $4.2 \%$; 26 events), gastrointestinal bleeding $(2.2 \%, 1 \cdot 4 \%$ to $3.4 \%$; 22 events), diarrhea $(1.9 \%, 1.2 \%$ to $3.0 \% ; 20$ events), ureteric strictures $(2.9 \%, 2.1 \%$ to $4.2 \%$; 36 events), urinary incontinence $(2.2 \%, 1.4 \%$ to $3.3 \% ; 24$ events), urinary frequency $(1.8 \%, 1.1 \%$ to $2.8 \% ; 19$ events), cystitis $(1.3 \%, 0.8 \%$ to $2.3 \% ; 14$ events), vaginal stenosis $(4.0 \%, 2.8 \%$ to $5.7 \% ; 36$ events), and vaginal mucositis $(1.4 \%, 0.8 \%$ to $2.3 \% ; 14$ events). In the post hoc analysis, fistulae and ureteric strictures were more common in 203 patients with 
stage IIIA-IIIB cancer (17 fistulae, $10.2 \%, 95 \% \mathrm{Cl} 6.3 \%$ to $16.2 \%$; 20 ureteric strictures, $10.8 \%, 6.9 \%$ to $16.8 \% ; \mathrm{n}=31$ patients), and in 34 patients with stage IVA cancer (six fistulae, $18.6 \%, 7.8 \%$ to $40.6 \%$; six ureteric strictures, $21.3 \%, 9.8 \%$ to $42.9 \%$; $n=12$ patients) than in 1005 patients with IB1-IIB cancer with 11 fistulae $(1.3 \%, 0.7 \%$ to $2.5 \%)$ and 10 ureteric strictures $(0.7 \%, 0.3 \%$ to $1.6 \% ; n=19$ patients). Furthermore, within the RetroEMBRACE and EMBRACE studies, organ-specific dose-effect relationships were addressed. For rectum, a dose for the most exposed $2 \mathrm{~cm}^{3}$ volume of $>65$ Gy results in $15 \%-25 \% \geq \mathrm{G} 2$ rectal morbidity, while when the dose is below $65 \mathrm{~Gy}$, the $\geq \mathrm{G} 2$ morbidity of this organ decreases to $5 \%-10 \%$. A dose for the most exposed $2 \mathrm{~cm}^{3}$ volume rectum $\geq 75$ Gy increases the risk for rectovaginal fistula. ${ }^{41}$ Bowel is a relatively new organ at risk for brachytherapy, introduced by the advent of image-guided adaptive brachytherapy. There is no clear delimitation of the small and large bowel, therefore the term 'bowel' refers to the rest of the large bowel minus the sigmoid and rectum and the small bowel close to the external beam radiation and brachytherapy target. ${ }^{24}$ The total severe toxicity on the bowel is limited. Actuarial overall G3,4 at $3 / 5$ years is $5 \% / 5.9 \%{ }^{42}{ }^{43}$ The bladder toxicity could be assessed by the dose to $2 \mathrm{~cm}^{3}$ of most exposed bladder volume and the International Commission on Radiation Units \& Measurements Bladder point. ${ }^{21}{ }^{31} 44$ Baseline urinary morbidity and overweight or obesity were significant risk factors for most endpoints. ${ }^{44}$ A dose $>80$ Gy to the most exposed $2 \mathrm{~cm}^{3}$ bladder volume results in $\geq \mathrm{G} 2$ toxicity in $30 \%-40 \%$ of patients, while below that dose the incidence decreases to $15 \%-30 \% .{ }^{45}$ The International Commission on Radiation Units \& Measurements bladder point doses $>75$ Gy increase the $\geq \mathrm{G} 2$ bladder incontinence. ${ }^{21}{ }^{44}$ The dose to the most exposed $2 \mathrm{~cm}^{3}$ bladder volume correlated with $\mathrm{G}>2$ fistula, bleeding and cystitis, while International Commission on Radiation Units \& Measurements bladder point dose correlated with pain. An increase from 75 Gy to 80 Gy resulted in an increase from $8 \%$ to $13 \%$ for the 4year actuarial estimate of $\mathrm{G}>2$ cystitis. ${ }^{44}$

A dose of $>75$ Gy to the International Commission on Radiation Units \& Measurements rectovaginal point increases the risk of $\geq \mathrm{G} 2$ vaginal stenosis. ${ }^{20}$ Currently, there is no dose-effect correlation regarding the specific sigmoid bowel morbidity or ureteral toxicity. However, for ureteral toxicity, we know that the overall incidence of ureteral stenosis following image-guided adaptive brachytherapy is quite limited (actuarial at 5 years $2.1 \%$ ), however in patients with hydronephrosis at the onset, the probability rises to $11.5 \%$. ${ }^{45} 46$

Moreover, based on the findings of EMBRACE, there are ongoing studies regarding the distinction between transient and persistent symptoms through Persistence of Late Substantial Patient-Reported Symptoms (LAPERS). ${ }^{47}$ Patient-reported symptoms (European Organization for Research and Treatment of Cancer (EORTC)-C30/CX24) and physician-assessed morbidity (Common Terminology Criteria for Adverse Events (CTCAE), version 3.0) were assessed at baseline and regular time points during follow-up. LAPERS events were defined as the presence of substantial EORTC symptoms (quite a bit/very much) for at least half of the assessments (persistence) and progression beyond the baseline condition (treatment related). For each EORTC symptom, the ratio between LAPERS rates and crude incidence rates of substantial symptoms was calculated to represent the proportion of symptomatic patients with persisting symptoms. For nine symptoms with a corresponding EORTC/ CTCAE assessment, the overlap of LAPERS and severe morbidity events (grades 3-5) was evaluated. Within a large cohort of survivors of locally advanced cervical cancer, a subgroup of patients with persistent symptoms (LAPERS events) was identified. These symptoms were diarrhea, blood in stool, difficulty in controlling the bowel, urinary frequency, urinary incontinence, lymphedema, insomnia, fatigue, and menopausal symptoms. For symptoms with a corresponding EORTC/CTCAE assessment, the vast majority of LAPERS events occurred in patients without corresponding severe physician-assessed morbidity. Across all symptoms, LAPERS rates were substantially lower than crude incidence rates, which emphasizes the importance of distinguishing between transient and persisting symptoms. ${ }^{47}$

Based on all these findings, the current EMBRACE II protocol ${ }^{24}$ recommends the following dose constraints for the target and the organs at risk ${ }^{17}$ (Table 1). However, when a case is faced in which dose constraints for organs at risk cannot be achieved, we recommend that the patient is counseled appropriately regarding the potential side effects prior to the application and the dose to target is not compromised. As mentioned before, potential strategies to improve an implant and to respect the dose constraints include the use of interstitial needles, and to spare organs at risk, vaginal loading de-escalation and attention to the applicator positioning. In cases of big tumors at the time of diagnosis or large tumor rest or progression at the time of brachytherapy, timely referral to a more experienced brachytherapy center should be considered.

\section{Future Studies}

Based on clinical evidence from RetroEMBRACE ${ }^{25}$ and EMBRACE I, ${ }^{17}$ 85 Gy EQD2 is being used as a hard dose constraint in the ongoing EMBRACE II study along with other multi-parametric dose prescriptions for targets and organs at risk for both external beam radiation and image-guided adaptive radiotherapy. Here, in addition to MRIguided adaptive brachytherapy, external beam radiation should be delivered through image guidance and by means of intensity modulated radiotherapy. The EMBRACE II study stopped accrual at the end of 2021. In parallel, new protocols, which incorporate the risk factor data and new systemic agents, will be developed for the next research questions which potentially could be answered by the next EMBRACE III studies.

\section{CONCLUSION}

The advent of image-guided adapted brachytherapy in addition to chemoradiotherapy has changed the practice due to its excellent results, which dramatically improve local and pelvic control with potential impact on the overall survival and diminished side effects. Based on these findings, MRI-guided adaptive brachytherapy is the new gold standard treatment of locally advanced cervical cancer, replacing the traditional two-dimensional point A concept. Imageguided adaptive brachytherapy has become the new standard of care in Europe according to the recommendations of the European Society of Gynaecologic Oncology, the European Society for Radiotherapy and Oncology, and the European Society of Pathology, ${ }^{48}$ and is incorporated in the American Society of Radiation Oncology cervical cancer guidelines. ${ }^{49}$

Contributors AS is the main author of the paper, performed the review and wrote the body of the paper and conclusions. JK wrote the introduction, prepared the figures and reviewed the whole paper. 
Funding The authors have not declared a specific grant for this research from any funding agency in the public, commercial or not-for-profit sectors.

Competing interests None declared.

Patient consent for publication Not applicable.

Ethics approval This study does not involve human participants.

Provenance and peer review Commissioned; internally peer reviewed.

\section{REFERENCES}

1 Director General calls for increased collaboration to fight cervical cancer at IAEA world cancer day event. Available: https://www. iaea.org/newscenter/news/director-general-calls-for-increasedcollaboration-to-fight-cervical-cancer-at-iaea-world-cancer-dayevent

2 Vale C, Tierney J, Stewart L. Concomitant chemoradiotherapy for cervical cancer: a systematic review and meta-analysis of individual patient data. Gynecol Oncol 2006;100:442-3.

3 Han K, Milosevic M, Fyles A, et al. Trends in the utilization of brachytherapy in cervical cancer in the United States. Int $J$ Radiat Oncol Biol Phys 2013;87:111-9.

4 Gill BS, Lin JF, Krivak TC, et al. National cancer data base analysis of radiation therapy consolidation modality for cervical cancer: the impact of new technological advancements. Int J Radiat Oncol Biol Phys 2014;90:1083-90.

5 Koom WS, Sohn DK, Kim J-Y, et al. Computed tomography-based high-dose-rate intracavitary brachytherapy for uterine cervical cancer: preliminary demonstration of correlation between dosevolume parameters and rectal mucosal changes observed by flexible sigmoidoscopy. Int J Radiat Oncol Biol Phys 2007;68:1446-54.

6 Pötter R, Dimopoulos J, Georg P, et al. Clinical impact of $\mathrm{MRI}$ assisted dose volume adaptation and dose escalation in brachytherapy of locally advanced cervix cancer. Radiother Oncol 2007;83:148-55.

7 Pötter R, Georg P, Dimopoulos JCA, et al. Clinical outcome of protocol based image (MRI) guided adaptive brachytherapy combined with 3D conformal radiotherapy with or without chemotherapy in patients with locally advanced cervical cancer. Radiother Oncol 2011;100:116-23.

8 van Dyk S, Narayan K, Bernshaw D, et al. Clinical outcomes from an innovative protocol using serial ultrasound imaging and a single MR image to guide brachytherapy for locally advanced cervix cancer. Brachytherapy 2016;15:817-24.

9 Nesvacil N, Schmid MP, Pötter R, et al. Combining transrectal ultrasound and CT for image-guided adaptive brachytherapy of cervical cancer: proof of concept. Brachytherapy 2016;15:839-44.

10 Schmid MP, Pötter R, Brader P, et al. Feasibility of transrectal ultrasonography for assessment of cervical cancer. Strahlenther Onkol 2013;189:123-8.

11 Mahantshetty U, Naga Ch P, Khadanga CR, et al. A prospective comparison of computed tomography with transrectal ultrasonography assistance and magnetic resonance imagingbased Target-Volume definition during image guided adaptive brachytherapy for cervical cancers. Int J Radiat Oncol Biol Phys 2018;102:1448-56.

12 Kidd EA, Siegel BA, Dehdashti F, et al. Clinical outcomes of definitive intensity-modulated radiation therapy with fluorodeoxyglucosepositron emission tomography simulation in patients with locally advanced cervical cancer. Int J Radiat Oncol Biol Phys 2010;77:1085-91.

13 Haie-Meder C, Pötter R, Van Limbergen E, et al. Recommendations from gynaecological (GYN) GEC-ESTRO Working Group (I): concepts and terms in 3D image based 3D treatment planning in cervix cancer brachytherapy with emphasis on MRI assessment of GTV and CTV. Radiother Oncol 2005;74:235-45.

14 Viswanathan AN, Thomadsen B, et al, American Brachytherapy Society Cervical Cancer Recommendations Committee. American brachytherapy Society consensus guidelines for locally advanced carcinoma of the cervix. Part I: general principles. Brachytherapy 2012;11:33-46.

15 Mahantshetty U, Poetter R, Beriwal S, et al. IBS-GEC ESTROABS recommendations for CT based contouring in image guided adaptive brachytherapy for cervical cancer. Radiother Oncol 2021;160:273-84.

16 Report 89. J Icru 2013;13.

17 Tanderup K, Nesvacil N, Kirchheiner K, et al. Evidence-based dose planning aims and dose prescription in image-guided brachytherapy combined with radiochemotherapy in locally advanced cervical cancer. Semin Radiat Oncol 2020;30:311-27.
18 Pötter R, Haie-Meder C, Van Limbergen E, et al. Recommendations from gynaecological (GYN) GEC ESTRO Working Group (II): concepts and terms in 3D image-based treatment planning in cervix cancer brachytherapy-3D dose volume parameters and aspects of 3D image-based anatomy, radiation physics, radiobiology. Radiother Oncol 2006;78:67-77.

19 Sturdza AE, Pötter R, Kossmeier M, et al. Nomogram predicting overall survival in patients with locally advanced cervical cancer treated with radiochemotherapy including image-guided brachytherapy: a Retro-EMBRACE study. Int J Radiat Oncol Biol Phys 2021;111:168-77.

20 Kirchheiner K, Nout RA, Lindegaard JC, et al. Dose-effect relationship and risk factors for vaginal stenosis after definitive radio(chemo)therapy with image-guided brachytherapy for locally advanced cervical cancer in the EMBRACE study. Radiother Oncol 2016;118:160-6.

21 Spampinato S, Fokdal LU, Pötter R, et al. Importance of the ICRU bladder point dose on incidence and persistence of urinary frequency and incontinence in locally advanced cervical cancer: an EMBRACE analysis. Radiother Oncol 2021;158:300-8.

22 Lindegaard JC, Fokdal LU, Nielsen SK, et al. Mri-Guided adaptive radiotherapy in locally advanced cervical cancer from a Nordic perspective. Acta Oncol 2013:52:1510-9.

23 Logsdon MD, Eifel PJ. Figo IIIB squamous cell carcinoma of the cervix: an analysis of prognostic factors emphasizing the balance between external beam and intracavitary radiation therapy. Int $J$ Radiat Oncol Biol Phys 1999;43:763-75.

24 Image guided intensity modulated external beam radiochemotherapy and MRI based adaptive brachytherapy in locally advanced cervical cancer (EMBRACE II). Available: https://www.embracestudy.dk/ UserUpload/PublicDocuments/EMBRACE\%20II\%20Protocol.pdf

25 Tanderup K, Fokdal LU, Sturdza A, et al. Effect of tumor dose, volume and overall treatment time on local control after radiochemotherapy including $\mathrm{MRI}$ guided brachytherapy of locally advanced cervical cancer. Radiother Oncol 2016;120:441-6.

26 Escande A, Khettab M, Bockel S, et al. Interaction between the number of chemotherapy cycles and brachytherapy Dose/Volume parameters in locally advanced cervical cancer patients. J Clin Med 2020;9. doi:10.3390/jcm9061653. [Epub ahead of print: 0106 2020].

27 Mahantshetty U, Gurram L, Bushra S, et al. Single application Multifractionated image guided adaptive High-Dose-Rate brachytherapy for cervical cancer: Dosimetric and clinical outcomes. Int J Radiat Oncol Biol Phys 2021;111:826-34.

28 Sturdza A, Pötter R, Fokdal LU, et al. Image guided brachytherapy in locally advanced cervical cancer: improved pelvic control and survival in RetroEMBRACE, a multicenter cohort study. Radiother Oncol 2016;120:428-33.

29 Pötter R, Tanderup K, Schmid MP, et al. MRI-guided adaptive brachytherapy in locally advanced cervical cancer (EMBRACE-I): a multicentre prospective cohort study. Lancet Oncol 2021;22:538-47.

30 Charra-Brunaud C, Salleron J, Menoux I. [Dose optimization in 3D pulsed dose rate brachytherapy for patients with locally advanced cervical cancer: A French multicenter phase II trial]. Cancer radiotherapie : journal de la Societe francaise de radiotherapie oncologique 2021.

31 Fokdal L, Sturdza A, Mazeron R, et al. Image guided adaptive brachytherapy with combined intracavitary and interstitial technique improves the therapeutic ratio in locally advanced cervical cancer: analysis from the retroEMBRACE study. Radiother Oncol 2016:120:434-40.

32 Kirisits C, Lang S, Dimopoulos J, et al. The Vienna applicator for combined intracavitary and interstitial brachytherapy of cervical cancer: design, application, treatment planning, and dosimetric results. Int J Radiat Oncol Biol Phys 2006;65:624-30.

33 Jürgenliemk-Schulz IM, Tersteeg RJHA, Roesink JM, et al. MriGuided treatment-planning optimisation in intracavitary or combined intracavitary/interstitial PDR brachytherapy using tandem ovoid applicators in locally advanced cervical cancer. Radiother Oncol 2009;93:322-30.

34 Mahantshetty U, Sturdza A, Naga Ch P, et al. Vienna-II ring applicator for distal parametrial/pelvic wall disease in cervical cancer brachytherapy: an experience from two institutions: clinical feasibility and outcome. Radiother Oncol 2019;141:123-9.

35 Keller A, Rodríguez-López JL, Patel AK, et al. Early outcomes after definitive chemoradiation therapy with Vienna/Venezia hybrid highdose rate brachytherapy applicators for cervical cancer: A singleinstitution experience. Brachytherapy 2021;20:104-11.

36 Gill BS, Kim H, Houser CJ, et al. Mri-Guided high-dose-rate intracavitary brachytherapy for treatment of cervical cancer: the University of Pittsburgh experience. Int J Radiat Oncol Biol Phys 2015;91:540-7. 


\section{Review}

37 Castelnau-Marchand P, Chargari C, Haie-Meder C, et al. Imageguided adaptive brachytherapy in locally advanced cervical cancer: recent advances and perspectives. Curr Opin Oncol 2016;28:419-28.

38 Eifel PJ, Morris M, Wharton JT, et al. The influence of tumor size and morphology on the outcome of patients with FIGO stage Ib squamous cell carcinoma of the uterine cervix. Int $J$ Radiat Oncol Biol Phys 1994;29:9-16.

39 Lanciano RM, Won M, Coia LR, et al. Pretreatment and treatment factors associated with improved outcome in squamous cell carcinoma of the uterine cervix: a final report of the 1973 and 1978 patterns of care studies. Int J Radiat Oncol Biol Phys 1991;20:667-76.

40 Fokdal L, Tanderup K, Hokland SB, et al. Clinical feasibility of combined intracavitary/interstitial brachytherapy in locally advanced cervical cancer employing MRI with a tandem/ring applicator in situ and virtual preplanning of the interstitial component. Radiother Oncol 2013;107:63-8.

41 Mazeron R, Maroun P, Castelnau-Marchand P, et al. Pulsed-dose rate image-guided adaptive brachytherapy in cervical cancer: Dosevolume effect relationships for the rectum and bladder. Radiother Oncol 2015;116:226-32.

42 K Jensen NB, Pötter R, Spampinato S, et al. Dose-volume effects and risk factors for late diarrhea in cervix cancer patients after radiochemotherapy with image guided adaptive brachytherapy in the embrace I study. Int J Radiat Oncol Biol Phys 2021;109:688-700.

43 Jensen NBK, Pötter R, Kirchheiner K, et al. Bowel morbidity following radiochemotherapy and image-guided adaptive brachytherapy for cervical cancer: physician- and patient reported outcome from the embrace study. Radiother Oncol 2018;127:431-9.

44 Spampinato S, Fokdal LU, Pötter R, et al. Risk factors and doseeffects for bladder fistula, bleeding and cystitis after radiotherapy with imaged-guided adaptive brachytherapy for cervical cancer: an embrace analysis. Radiother Oncol 2021;158:312-20.

45 Fokdal L, Pötter R, Kirchheiner K, et al. Physician assessed and patient reported urinary morbidity after radio-chemotherapy and image guided adaptive brachytherapy for locally advanced cervical cancer. Radiother Oncol 2018;127:423-30.

46 Fokdal L, Tanderup K, Pötter R, et al. Risk factors for ureteral stricture after radiochemotherapy including image guided adaptive brachytherapy in cervical cancer: results from the embrace studies. Int J Radiat Oncol Biol Phys 2019;103:887-94.

47 Vittrup AS, Tanderup K, Bentzen SM, et al. Persistence of late substantial patient-reported symptoms (LAPERS) after radiochemotherapy including image guided adaptive brachytherapy for locally advanced cervical cancer: a report from the embrace study. Int J Radiat Oncol Biol Phys 2021;109:161-73.

48 Cibula D, Pötter R, Planchamp F, et al. The European Society of Gynaecological Oncology/European Society for Radiotherapy and Oncology/European Society of Pathology guidelines for the management of patients with cervical cancer. Int J Gynecol Cancer 2018;28:641-55.

49 Chino J, Annunziata CM, Beriwal S, et al. The ASTRO clinical practice guidelines in cervical cancer: optimizing radiation therapy for improved outcomes. Gynecol Oncol 2020;159:607-10. 